\title{
A New Deformation Pose Estimation Algorithm for the Fully Automatic Design of Patient Specific Knee Prosthesis: Preliminary Results
}

\author{
Charles Garraud ${ }^{1}$, Arnaud Clavé ${ }^{1,2}$, Jérôme Ogor $^{1}$, Eric Stindel ${ }^{1,3,4}$, and \\ Guillaume Dardenne $e^{1,3}$ \\ ${ }^{1}$ LaTIM UMR1101 INSERM, Brest, France \\ ${ }^{2}$ Clinique Saint George, Nice, France \\ ${ }^{3}$ Brest University Hospital Center, Brest, France \\ ${ }^{4}$ University of Western Brittany, Brest, France \\ guillaume.dardenne@univ-brest.fr
}

\begin{abstract}
In the context of automatic landmarks localization with statistical shape models for the design of customized TKA prosthesis, the first step consists of registering a model, represented by the mean mesh of some healthy femoral bones, towards the segmented femur of the patient. The most complex aspect of the mesh-to-mesh correspondence in this case lies in the fact the source (model) and the target mesh can differ largely (partial view of the femur, anatomy that lies away from the mean) which makes common correspondence approaches inefficient. In this paper, we introduce a contribution to an algorithm from the field of object recognition that produces a reliable registration. By adding the concept of global deformability in the algorithm, we are able to improve the precision of the algorithm (mean mesh-to-mesh distance improved from $2.77 \mathrm{~mm}$ to $0.79 \mathrm{~mm}$ ) and its robustness to anatomy far off the mean (better standard deviation and Hausdorff distance) on synthetic data. The next step will be to assess it in its application field i.e. the automatic localization of knee landmarks for the design of patient-specific knee prosthesis.
\end{abstract}

\section{Introduction}

Customized TKA implants aim at improving accuracy, functional results and finally cost of total knee replacement surgery (Hafez and Moholkar, 2017)(Schwechter and Fitz,2012). In such procedures, 
disparity between patient anatomy and knee implant geometry may result in choosing the «least worse compromise» and thus negatively influence post-operative result ( $\mathrm{Li}$ et al., 2019). The pre-operative automatic planning workflow under development consists of the deformation of a template implant from anatomical landmarks and measurements (anatomical parameters) according to pre-defined mechanical constraints. Some of the methods considered in the past to automatically localize regions of interest in the knee include learning-based (Xue et al., 2015), CNN-based (Yang et al., 2015) or atlas-based (Mahfouz et al., 2009) procedures. We believe an approach based on statistical shape model(SSM) would be beneficial as it has been shown for premorbid anatomy prediction in shoulder surgery (Salhi et al., 2019) (Abler et al., 2018).

The selected procedure depends on the deformation of a SSM created from healthy femur bones towards the current anatomy of the patient. The success of this process relies on a nice initial rigid correspondence between the SSM (commonly presented by the mean of the femur bones) and the patient segmented femur. This kind of mesh-to-mesh correspondences is well studied (Guan et al., 2018) but the common approaches will eventually fail in our scenario due to the following issues:

1. The segmented femur could only be a partial view of the femur anatomy depending on the imaging output.

2. The process must be fully automatic and thus, robust to far-off position, flip and able to detect bone side (right or left).

3. The patient anatomy may vary largely from the SSM mean making the source mesh and target mesh being potentially very different in terms of shape.

Lots of our constraints are also experienced in the object recognition field that addresses the problem of recognizing free-form objects in points cloud. Point-pair features based algorithms as described in (Drost et al., 2010) tackle the first two issues nicely but the last one remains unsolved. In this paper, we propose a contribution to an existing pose estimation algorithm that accounts for all the above issues.

\section{Material and Methods}

\subsection{Statistical Shape Model}

The statistical shape model was generated from 20 cadaveric femurs scanned at CHRU Brest including 12 right and 8 left femur bones (Asvadi et al., 2019).

\subsection{Framework}

For the purpose of the study, an applicative framework (Tailor) was developed in $\mathrm{C}++$. The deformation material relies on Statismo (Lüthi et al., 2012) and a planning application (KneeSuite) is included.

\subsection{Processing pipeline}

The algorithm is based on the ones described in (Drost et al., 2010) and (Vidal et al., 2018) that compares point-pair features (PPF) from an object to point-pair features from a scene in order to select the best transformation according to a voting scheme. With our contribution, the algorithm is now able to account for the deformability of the object to be detected by considering a set of possible global deformation described by the SSM as input. With a single deformation model, the wrong pair can be selected if the pair representing the true anatomical match is too different from the mean shape on the 
patient segmented femur. Using different deformations as input solves the issue. The algorithm computes the best transformation as well as the best deformation from the initial ones as output.

\subsection{Validation}

The preliminary validation pipeline consists of registering and deforming the femur SSM towards synthetic data with a first run not accounting for deformation and a second run that accounts for 18 different deformations. The synthetic data used for the validation are computed by randomly deforming, sideflipping and space transforming the femur reference shape of the SSM. For the second run, the 18 deformations (9 left and 9 right) account for three different variations of the two principal deformation modes of the SSM. The validation pipeline involves the initial rigid registration step performed by our PPF algorithm followed by the deformation step described above. The precision is then assessed by computing distance measurements (mean distance, Hausdorff distance and RMS distance) between the registered mesh and the synthetic input data.

\section{Results}

The distance measurements are presented in Table 1. Accounting for deformations (Run 2) improves all measurements. There is a significant decrease of the mean distance mean from $2.77 \mathrm{~mm}$ to $0.79 \mathrm{~mm}$. The mean standard deviation is also reduced from $1.87 \mathrm{~mm}$ to $0.68 \mathrm{~mm}$. All maximum measurements (Hausdorff) are improved by more than $70 \%$.

\begin{tabular}{|c|c|c|c|c|c|c|c|c|c|}
\hline & \multicolumn{3}{|c|}{ Mean (mm) } & \multicolumn{3}{|c|}{ RMS } & \multicolumn{3}{|c|}{ Hausdorff (mm) } \\
\hline & $\begin{array}{l}\text { Mean } \\
\text { (SD) }\end{array}$ & Min & Max & $\begin{array}{c}\text { Mean } \\
\text { (SD) }\end{array}$ & Min & Max & $\begin{array}{l}\text { Mean } \\
\text { (SD) }\end{array}$ & Min & Max \\
\hline Run 1 & $\begin{array}{c}2.8 \\
(1.9)\end{array}$ & 0.6 & 12.0 & $\begin{array}{c}4.5 \\
(2.9)\end{array}$ & 0.8 & 17.0 & $\begin{array}{c}17.4 \\
(11.3)\end{array}$ & 3.1 & 61.0 \\
\hline Run 2 & $\begin{array}{c}0.8 \\
(0.7)\end{array}$ & 0.2 & 3.7 & $\begin{array}{c}1.0 \\
(0.9)\end{array}$ & 0.3 & 4.4 & $\begin{array}{c}3.8 \\
(3.9)\end{array}$ & 0.9 & 18.3 \\
\hline
\end{tabular}

Table 1: Distance measurement results

\section{Discussion and further work}

We presented a new algorithm for the initial correspondence of a SSM with a patient segmented femur. The algorithm, based on robust object-recognition algorithms (Drost et al., 2010)(Vidal et al., 2018) accounts for the deformability of the SSM in the initial pose computation. It shows promising results in term of robustness on synthetic data. Accounting for deformability definitely improves the precision of the registration. A further analysis of the enhancement in terms of standard deviation, maximum distances and Hausdorff distances shows that the algorithm behaves especially well to far off the mean inputs thus greatly boosting the performances in these extreme cases. The next step consists of a validation of its use in the localization of landmarks which will be used for the design of patient-specific knee implants. 


\section{Acknowledgement}

This work benefited from State aid managed by the National Research Agency under the future investment program bearing the reference ANR-17-RHUS-0005.

\section{References}

Abler, D., Berger, S., Terrier, A., Becce, F., Farron, A., and Büchler, P. (2018). A statistical shape model to predict the premorbid glenoid cavity. Journal of Shoulder and Elbow Surgery, 27.

Asvadi, A., Dardenne, G., Guezou-Philippe, A., Salhi, A., Borotikar, B., Troccaz, J., and Burdin, V. (2019). Kernel selection in statistical femur modeling. https://hal.archivesouvertes.fr/hal-02183893.

Drost, B., Ulrich, M., Navab, N., and Ilic, S. (2010). Model globally, match locally: Efficient and robust 3d object recognition. Proceedings of the IEEE Computer Society Conference on Computer Vision and Pattern Recognition, pages 998-1005.

Guan, S.-Y., Wang, T.-M., Meng, C., and Wang, J.-C. (2018). A review of point feature based medical image registration. Chinese Journal of Mechanical Engineering, 31 .

Hafez, M. and Moholkar, K. (2017). Patient-specific instruments: advantages and pitfalls. SICOT-J, 3:66.

Li, K., Saffarini, M., Valluy, J., Desseroit, M.-C., Morvan, Y., No, T., and Cavaignac, E. (2019). Sexual and ethnic polymorphism render prosthetic overhang and under-coverage inevitable using offthe shelf tka implants. Knee Surgery, Sports Traumatology, Arthroscopy, 27.

Lüthi, M., Albrecht, T., Gass, T., Goksel, O., Kistler, M., Bousleiman, H., Reyes, M., Büchler, P., Cattin, P., and Vetter, T. (2012). Statismo - a framework for pca based statistical models. The Insight Journal, 1:1-18.

Mahfouz, M., Abdel Fatah, E., Merkl, B., and Mitchell, J. (2009). Automatic and manual methodology for three-dimensional measurements of distal femoral gender differences and femoral component placement. The journal of knee surgery, 22:294-304.

Salhi, A., Burdin, V., Boutillon, A., Brochard, S., Mutsvangwa, T., and Borotikar, B. (2019). Statistical shape modeling approach to predict missing scapular bone. Annals of Biomedical Engineering, 48.

Schwechter, E. and Fitz, W. (2012). Design rationale for customized tka: A new idea or revisiting the past. Current reviews in musculoskeletal medicine, 5.

Vidal, J., Lin, C.-Y., Llado, X., and Mart'1, R. (2018). A method for 6 d pose estimation of free-form rigid objects using point pair features on range data. Sensors, 18:2678.

Xue, N., Döllinger, M., Ho, C., Surowiec, R., and Schwarz, R. (2015). Automatic detection of anatomical landmarks on the knee joint using mri data. Journal of magnetic resonance imaging: JMRI, 41.

Yang, D., Zhang, S., Yan, Z., Tan, C., Li, K., and Metaxas, D. (2015). Automated anatomical landmark detection on distal femur surface using convolutional neural network. volume 2015. 